\title{
A decrease of $\geqslant 2$ points on the Mini-Mental State Examination was the best determinant of delirium in elderly patients admitted to hospital
}

O'Keeffe ST, Mulkerrin EC, Nayeem K, et al. Use of serial Mini-Mental State Examinations to diagnose and monitor delirium in elderly hospital patients. J Am Geriatr Soc 2005;53:867-70.

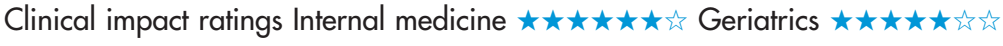

In elderly patients admitted to hospital, are serial Mini-Mental State Examinations (MMSEs) accurate for diagnosing delirium?

\section{METHODS}

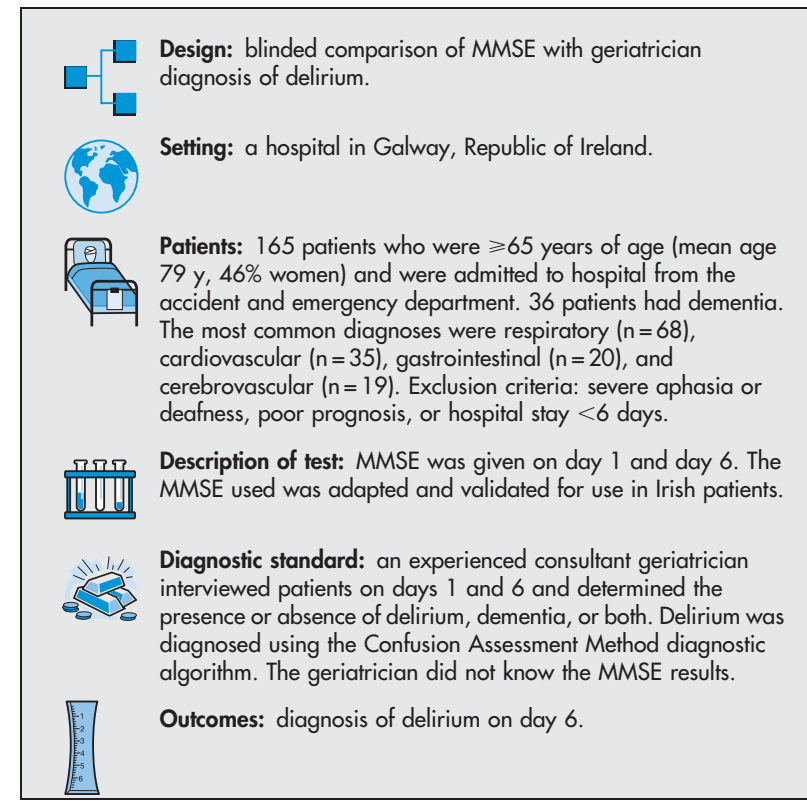

\section{MAIN RESULTS}

138 patients were not delirious at admission. On day 6, 14 of these patients had delirium. A decrease of $\geqslant 2$ points on the MMSE was the best determinant for detecting delirium (area under the receiver operating characteristic curve $0.97,95 \%$ CI 0.95 to 1.00 ). The table shows the diagnostic characteristics.

\section{CONCLUSION}

In elderly patients admitted to hospital, a decrease of $\geqslant 2$ points on the Mini-Mental State Examination was the best determinant of delirium.

\section{Commentary}

D elirium frequently accompanies acute medical illness among hospitalised patients. Characteristic features include acute onset and fluctuations in severity over time. Average duration is about 6 days, ${ }^{1}$ although for some patients the impairment may persist longer. Typically, delirious patients are older, frailer, and more likely to have baseline cognitive and physical impairments. ${ }^{2}$ Delirium is related to several adverse outcomes, including longer mean length of hospital stay, poorer functional status, greater likelihood of institutionalisation, and higher mortality, which may be sustained over 12 months. ${ }^{13}$ Prognostic indicators for poor outcome include baseline frailty, absence of agitation, and under-recognition of delirium. ${ }^{1}$ Busy clinicians require simple instruments to quickly recognise the syndrome.

The study by O'Keeffe et al shows the utility of repeated MMSE measurements in vulnerable, hospitalised, elderly patients. A decrease of $\geqslant 2$ points was predictive of the development of delirium; an increase of $\geqslant 3$ points was associated with resolution. Mean change of MMSE scores in those who worsened or improved was -4.5 and 4.8 , respectively. In patients with baseline dementia, frequent MMSEs can help determine who has concomitant dementia on admission and who develops delirium based on a rise or fall in MMSE of 4-5 points. However, a 6 day interval between tests may be too long. A low index of clinical suspicion with more frequent MMSEs may improve the utility of this method for diagnosing delirium. Another tool that has been used to monitor fluctuations over time is the Delirium Index, which may be more useful than the MMSE alone in patients with delirium. ${ }^{4}$

\section{Suzanne D Fields, MD
SUNY Stony Brook School of Medicine} Stony Brook, New York, USA

1 Andrew MK, Freter SH, Rockwood K. Incomplete functional recovery after delirium in elderly people: a prospective cohort study. BMC Geriatr 2005;5:5.

2 Korevaar JC, van Munster BC, de Rooij SE. Risk factors for delirium in acutely admitted elderly patients: a prospective cohort study. BMC Geriatr 2005:5:6.

3 McCusker J, Cole M, Abrahamowicz M, et al. Delirium predicts 12-month mortality. Arch Intern Med 2002;162:457-63.

4 McCusker J, Cole MG, Dendukuri N, et al. The delirium index, a measure of the severity of delirium: new findings on reliability, validity, and responsiveness. J Am Geriatr Soc 2004;52:1744-9.
For correspondence: Dr S O'Keeffe, Galway Regional Hospitals, Galway, Ireland. S.okeeffe@whb.ie

Source of funding: no external funding.

Test characteristics for detecting delirium using a decrease of $\geqslant 2$ points on the Mini-Mental State Examination*

\begin{tabular}{llll}
\hline Sensitivity & Specificity & + LR $(95 \%$ CI) & - LR \\
\hline $93 \%$ & $90 \%$ & $8.9(5.2$ to 15.1$)$ & 0.08 (0.01 to 0.53$)$ \\
\hline *Diagnostic terms defined in glossary. & &
\end{tabular}

